\title{
OMISSION OU EXCLUSION ? \\ MARCELLE TINAYRE ET LE CANON LITTÉRAIRE
}

France GRENAUDIER-KLIJN, Université Massey

\section{Résumé}

À la croisée des $\mathrm{XIX}^{\mathrm{e}}$ et $\mathrm{XX}^{\mathrm{e}}$ siècles, Marcelle Tinayre (1870-1948) fut une romancière à la fois lue par un large public et respectée par la critique et par ses pairs. Néanmoins, il est rare que son nom apparaisse dans les anthologies littéraires consacrées à la période. Auteure de plus de vingt-deux textes, récompensée par plusieurs prix, pourquoi cette écrivaine compte-t-elle aujourd'hui au nombre des grandes oubliées de l'histoire littéraire française ? C'est sur cette interrogation que s'appuiera la discussion qui suit.

Ancrée dans une perspective féministe étayée par la notion de «violence symbolique », au sens où la définissent Pierre Bourdieu et Jean-Claude Passeron, la présente étude s'interroge sur les raisons pour lesquelles l'histoire littéraire d'une nation, en l'occurrence la France, inscrit tel/le auteur/e à son panthéon et en néglige tel/le autre. Après un bref rappel des conditions particulières instaurées par l'identité auctoriale au féminin, et des présupposés présidant à l'élaboration du canon littéraire en général, nous nous pencherons sur les circonstances propres à l'avènement de Marcelle Tinayre en tant que femme de lettres française. Pour ce faire, nous rappellerons tout d'abord le climat idéologique ayant entouré la gestation, la diffusion et la réception de ses textes, facteurs hétérogènes ayant en commun de se situer très largement hors de la sphère d'influence de l'auteure. Dans un deuxième temps, nous examinerons les facteurs dits endogènes, c'est-à-dire les éléments dépendants de l'agir même de Tinayre : créativité, originalité, thématiques, prises de position. Nous conclurons en formulant quelques hypothèses susceptibles d'expliquer l'absence de l'écrivaine et de son œuvre au canon littéraire français, et en nous interrogeant sur la légitimité et la validité du processus de canonisation.

\section{Introduction}

En 1934, quatorze ans avant la mort de Marcelle Tinayre, le critique Pierre Trintignac écrivait dans Le Courrier du centre : 
Il est probablement assez vain d'anticiper sur le jugement de la postérité en s'inquiétant de ce qu'elle pourra retenir de la littérature contemporaine. Les écrits qui nous enchantent laisseront peut-être indifférent le lecteur de l'an 2000, et ce lecteur trouvera sans doute dans les pages les plus inattendues des beautés que nous n'aurons même pas soupçonnées. Cependant, pour le cas particulier de $M^{\text {me }}$ Tinayre, nous ne croyons pas qu'on puisse oublier sans injustice ces livres humains et forts qui s'appellent La Maison du péché, La Vie amoureuse de François Barbazanges, La Rebelle et L'Ennemie intime. (n. p.)

Marcelle Tinayre, née en 1870 à Tulle, morte en 1948 à Montfort L'Amaury, auteure de plus de vingt romans, très populaire en son temps, est aujourd'hui largement oubliée. Son nom n'apparaît dans aucune anthologie d'importance. Même si plusieurs études lui ont été consacrées, sous la forme d'articles savants ou de monographies ${ }^{1}$, sa renommée reste confinée aux cercles universitaires. Cet oubli est-il mérité ou s'agit-il, comme le suggérait Trintignac, d'une injustice?

Autant le dire tout de suite, je ne suis pas certaine de pouvoir répondre de manière objective à cette question. Je ne cherche pas non plus à réhabiliter cette auteure (en admettant que cela soit possible), pas plus que je n'ai l'intention d'exhumer son œuvre. Ce qui m'intéresse, c'est de cerner les facteurs susceptibles d'avoir mené l'œuvre de Marcelle Tinayre à l'oubli. Ces facteurs, je les diviserai globalement en deux catégories. Il y d'abord des raisons que je qualifierai d'hétérogènes, au sens où celles-ci sont périphériques à l'œuvre et relèvent des mécanismes de diffusion, des critères taxinomiques, et plus généralement d'un contexte socioculturel et politique sur lequel la romancière n'a que peu d'influence. J'appellerai les autres des facteurs endogènes ayant partie liée aux particularités thématiques et stylistiques de l'écriture tinayrienne, ainsi qu'aux engagements et prises de position de l'auteure. Bien évidemment, ces données se recoupent, et c'est la conjonction des facteurs politiques, idéologiques, socioculturels, littéraires et biographiques qui ensemble contribuent à la mise au rebut, ou non, d'un/e écrivain/e. Il s'agit donc d'examiner le rôle joué par l'intervention conjointe des paramètres «externes» et «internes» dans la réception et la pérennisation de l'œuvre tinayrienne, et de souligner la nécessité qu'il y a à en tenir concomitamment compte. Je m'accorde ainsi avec le postulat adopté par Pierre Bourdieu qui rejette catégoriquement l'anhistoricisme prôné par l'herméneutique (post)structuraliste et dénonce ceux qui, tel Michel Foucault, prétendent à l'autonomie absolue de l'ordre culturel. Indépendamment des souhaits exprimés par les «esthétiques formalistes » 
(78), expliquait Bourdieu lors d'une conférence prononcée à Princeton University en 1986, le microcosme littéraire est mû par «des instruments et des enjeux de lutte » (66). Le nier serait non seulement naïf mais captieux. Il me semble dès lors salutaire, voire honorable, d'aborder l'histoire littéraire avec circonspection, en nous souvenant que la constitution du canon s'engendre au sein d'un faisceau de conceptions stylistiques, thématiques, génériques - et de présupposés - économiques, politiques, idéologiques - qui compromettent sérieusement toute prétention à l'objectivité comme à l'exhaustivité.

De plus, nous avons ici affaire à une femme de lettres, ce qui nous impose d'ajouter une dimension genrée aux remarques qui précèdent. Celles-ci en effet pourraient tout aussi bien s'appliquer aux coreligionnaires masculins de Marcelle Tinayre, les nombreux oubliés et exclus de l'histoire littéraire. Or, il est indubitable que le capital culturel, économique, social et symbolique de Tinayre (qui déterminent et relèvent de son habitus, selon la terminologie bourdieusienne) a été affecté par les conditions particulières instaurées par son identité auctoriale, ces mêmes conditions influant à leur tour sur le processus de canonisation. Comme l'explique Rotraud von Kulessa dans l'introduction de l'ouvrage qu'elle vient de consacrer aux «autrices » dans les champs littéraires français et italien des années 1900 : «[étant donné] la quantité et la richesse de la production littéraire de femmes [à l'époque 1900], la question de savoir pourquoi celles-ci ne sont guères présentes dans les manuels d'histoire littéraire ou tout simplement dans la mémoire collective se fait de plus en plus pressante » (20). Ceci me semble même relever d'une véritable nécessité car l'on ne saurait comprendre certains des mécanismes ayant régi et continuent dans une certaine mesure à régir le champ littéraire français sans tenir compte de l'aporie constituée par l'omission d'un très grand nombre de romancières dans les exégèses et les anthologies. Sans pour autant nous adonner à une interprétation strictement historiciste, il reste clair qu'il doit être tenu compte des diverses limitations imposées à Tinayre en tant que femme de lettres à tous les stades du processus de création littéraire : gestation, production, promotion, diffusion, réception, consécration, pérennisation. Ce n'est qu'ainsi, me semble-t-il, que nous serons en mesure d'appréhender et de comprendre le hiatus constitué par l'exclusion de son œuvre par le canon. Enfin, si nous poussons plus loin l'argument, nous devons nous demander si l'oubli imposé au corpus tinayrien, voire à l'œuvre de nombre de romancières de la période, ne relève pas d'une forme diffuse de ce que Pierre Bourdieu et Jean-Claude 
Passeron appellent la «violence symbolique» qu'ils définissent comme «tout pouvoir qui parvient à imposer des significations et à les imposer comme légitimes en dissimulant les rapports de force qui sont au fondement de sa force » (2). Je reviendrai sur ce point dans ma conclusion.

\section{Le canon}

Commençons par le canon littéraire : sur quelles bases s'établit-il ? À quels critères obéit-il ? À quelles exigences répond-il ? De manière très schématique, on s'accorde sur le fait que le canon littéraire réunit des œuvres jugées dignes de passer à la postérité de par leurs qualités littéraires exceptionnelles. Mais, outre qu'on ne saurait négliger la petite part de chance susceptible d'entrer en jeu dans la détermination du canon - il arrive en effet qu'un hasard bienheureux nimbe une œuvre et ouvre à son auteur/e les portes de la pérennité - l'activité littéraire est en réalité toujours-déjà socialement et culturellement médiatisée, autant au niveau de sa production, qu'à celui de sa diffusion, et à celui de sa consécration. Ce processus de création ne s'effectue pas ex nihilo mais s'insère dans un subtil réseau d'influences où interviennent des facteurs littéraires - réécritures, emprunts - et des facteurs horstextuels. De plus, les caractéristiques stylistiques, structurelles, idéologiques et thématiques des œuvres écrites - les œuvres orales peinant davantage à trouver place dans le canon - se modifient avec le temps, tout comme varient les conceptions artistiques et les valeurs idéologiques des éditeurs, de l'appareil critique et médiatique, et des lecteurs. C'est ce qui fait par exemple que des écrivains populaires en leur temps soient ultérieurement jugés « démodés ». Il arrive même que ces affects se surimposent au mérite purement littéraire d'une œuvre. Pensons ici à la controverse récemment survenue en France à l'occasion du cinquantenaire de la mort de Céline ${ }^{2}$.

Si les anthologies de littérature du type Lagarde \& Michard semblent avoir pour fonction essentielle de refléter l'évolution de la création littéraire au fil des genres - théâtre, poésie, roman - et des époques, ce n'est pas tout à fait, pas entièrement, pas exclusivement, le cas. Ces textes sont triés, sélectionnés, organisés, agencés et hiérarchisés «en fonction d'un consensus social [tacite] » (André 110111). L'inclusion ou l'exclusion d'une œuvre au patrimoine culturel et institutionnel n'a donc rien d'aléatoire. Si le canon prétend résulter d'un travail d'appréciation engagé en vertu de critères esthétiques donnés comme objectifs, il y fait en réalité intervenir également des partis-pris axiologiques qui eux aussi se colorent selon 
l'humeur idéologique de l'espace socioculturel en question. Le canon correspond dès lors à un processus d'institutionnalisation et de légitimation résultant non pas d'une approche empirique et/ou épistémologique «pure» mais d'un «paradigme qui recompose la réalité selon ses normes, au nom de ce que les choses devraient être » (Chardenet 9). De la sorte, le modus operandi du système canonical illustre de manière très convaincante le concept d'habitus théorisé par Pierre Bourdieu, dans la mesure où son dispositif repose sur des pratiques et des mécanismes prédéterminés et s'établit à partir de lectures et de questionnements interprétatifs eux-mêmes tributaires d'un ensemble de jugements esthétiques subjectifs. En d'autres termes, le canon se construit sur des «structures structurées disposées à fonctionner comme structures structurantes » (Bourdieu, Sens, 88-89). On ne peut donc nier que la (re)production du canon, en tant que dispositif et en tant que système, recèle un potentiel politique conséquent, dès lors que son objectif s'avère, in fine, le maintien de la légitimité supériorité ? - culturelle d'un groupe d'agents sociaux. Parallèlement, à partir du moment où l'on tient compte des processus d'exclusion, de hiérarchisation et de différenciation inhérents au dispositif canonical, il est difficile de ne pas y voir la manifestation d'une certaine modalité de pouvoir susceptible d'œuvrer à une forme de domination, d'autorité, voire de violence symbolique.

Différentes instances de pérennisation - archives, bibliothèques, anthologies, comptes rendus, critiques - vont ainsi apporter leur pierre à l'édification du patrimoine littéraire d'une nation. Or, comme le rappelle Martine Lavaud, «toute histoire littéraire, en tant que construction rétrospective, est une fiction » (163), d'où il ressort que tout canon littéraire est, par définition, subjectif et lacunaire. L'on pourrait fort bien s'accommoder de cette nature fragmentaire si l'on ne négligeait pas du même coup de souligner le caractère naturellement assujettissant du canon. Or, tel n'est pas le cas. À cet égard, le rôle de l'école est particulièrement important, puisque c'est dans le champ pédagogique que vont être enseignées les œuvres retenues et présentées comme exemplaires (aux deux sens du terme), dans un objectif édificateur, mais aussi - on l'oublie parfois - «indéniablement politique » (André 110). Le champ pédagogique français ayant pour principal objectif de «former les formateurs de la République [et] d'asseoir dans ses grandes lignes l'idéologie nationale » (110), il n'est pas étonnant d'assister, dans les cours et les manuels, à la disparition de pans entiers de la production littéraire nationale jugés «marginales », telles, par exemple, les littératures populaires, régionales, et, bien souvent, féminines. 


\section{Les femmes et le patrimoine littéraire}

Que retient l'histoire littéraire d'une nation? Très schématiquement, les hommes. Quoique l'on ne saurait négliger le poids des préjugés «gaulois » - il n'est pas anodin, à cet égard, que la majorité des travaux consacrés à Tinayre n'aient pas été publiés en France, mais en Italie, au Royaume-Uni, en Nouvelle-Zélande, aux États-Unis ou encore au Canada - et même si l'arène littéraire française reste un bastion masculin particulièrement réticent à prendre en considération les ouvrages rédigés par des femmes, toutes périodes confondues ${ }^{3}$, ce phénomène n'est pas unique à la France. Outre qu'elle persiste, dans son introduction, à vouer une section aux «women writers », ce qui montre à quel point nous ne sommes pas encore sortis d'un certain mode de conceptualisation de la production littéraire, la toute récente Cambridge History of French Literature, qui ne cache pas ses ambitions de devenir $\mathrm{LA}^{4}$ référence en matière de littérature française, ne mentionne quasiment aucune auteure pour la période qui nous intéresse, c'est-à-dire grosso modo les années 1890 à 1930. Cette omission s'avère d'autant plus surprenante quand on pense au nombre de romancières en activité à l'époque - en 1894, Octave Uzanne avait dénombré 1219 femmes inscrites à la Société des gens de lettres ${ }^{5}$. Difficile de croire qu'elles étaient toutes lamentables ! Mais il est vrai que la tradition occidentale a souvent eu tendance à «personnaliser» les auteurs/es, ce qui a pu conduire l'identité sexuelle à opérer dans une logique ambiguë : outil promotionnel et/ou instrument de dévalorisation. Dans les années qui nous occupent, les romancières eurent ainsi bien du mal à se défaire des accusations de «bas-bleuisme» et autres dénigrements, les écrivaines ayant «toujours été [considérées] femmes avant d'être écrivains » (Slama 52). Or, l'excessive importance accordée alors à l'appartenance genrée des auteures réduisait considérablement leur champ de manœuvres à toutes les étapes du processus littéraire.

Ainsi, l'essor considérable des œuvres féminines durant la période a encouragé l'homologie et contribué à une forme d'anonymat qui a, à son tour, facilité l'oubli. En effet, la très grande majorité des romancières de cette époque ont été noyées dans la masse, à l'exception de Colette et, dans une moindre mesure, d'Anna de Noailles (et pas forcément pour des motifs purement littéraires) et sont passées dans les poubelles de l'histoire et de la culture. Alors que nombre de critiques semblèrent ébaubis par la multiplicité des parutions dues aux femmes - André Billy parla d' «explosion» (230); Henriette Charasson d' «apparition foudroyante » (65) ces commentateurs n'eurent de cesse de déprécier la qualité des textes en question. Ce 
pseudo-émerveillement face à un surenchérissement purement quantitatif a eu deux conséquences négatives pour les écrivaines de l'époque : il a d'une part compromis leurs chances de se singulariser, et d'autre part il a contribué à la dévalorisation systématique de leurs œuvres présentées comme un phénomène de mode, c'est-à-dire comme une sorte de convulsion superficielle et éphémère sans grand mérite. Tinayre en était d'ailleurs parfaitement consciente. Voici ce qu'elle déclarait à une journaliste en 1922: «Certes les femmes ont du talent, mais pourquoi toujours les comparer entre elles? On nous laisse dans notre cage aux singes ensemble et c'est une manière de nous prouver qu'on ne met pas notre talent à hauteur de celui des hommes. Cela dites-le, mademoiselle, c'est une injustice » (Havet n. p.). Certes, le canon ne peut pas citer toutes les œuvres; c'est impossible - manque de place - et surtout cela va à l'encontre du projet canonical qui est de retenir les œuvres d'exception. Mais, il est clair que le discrédit imposé à la production romanesque féminine française des années 1890-1930 résulta d'un mécanisme foncièrement pernicieux consistant à reprendre d'un côté ce qu'on venait de « donner » de l'autre.

\section{Une difficile catégorisation}

Outre les limitations résultant de l'identité genrée de son auteure, trois facteurs échappant très largement à la sphère d'influence de la romancière viennent compliquer le processus taxinomique, diminuant d'autant les chances d'accès au canon. Il faut tout d'abord signaler que la période à laquelle paraissent les romans de Tinayre correspond à la croisée des dix-neuvième et vingtième siècles. Il résulte de cet entre-temps qu'il est difficile, y compris aux spécialistes, de rattacher l'œuvre à une période historique donnée. Or, compte tenu des modes de fonctionnement et d'élaboration du canon, qui privilégient les cloisonnements stricts, les divisions simples par écoles, périodes, genres, registres - comme le fait d'ailleurs toujours la Cambridge History of French Literature précédemment citée - ce «flou » temporel n'a pu que desservir l'écrivaine. Immédiatement précédées par les symbolistes, les naturalistes et les décadents, puis suivies par une nouvelle génération d'écrivains, tels que Claudel, Gide, Proust ou encore Bernanos, Tinayre et ses consœurs ont eu la malchance d'émerger dans une période difficile à cerner, sinon sous le label inepte de «roman féminin », ce qui, on s'en doute, ne favorisa pas leur entrée dans les annales.

Parallèlement, les origines sociales de Tinayre ont à leur tour amoindri ses chances de marquer de manière durable le champ culturel de son temps et, a fortiori, 
son inscription au patrimoine littéraire français. Fille de ce que Bourdieu décrit comme la petite bourgeoisie d'exécution - sa mère, Louise Chasteau, fut institutrice puis directrice d'école - Marcelle Tinayre bénéficie d'un indéniable capital culturel le jour où elle passe son bac en 1888 elles ne sont que deux filles - mais est dépourvue du capital social et économique nécessaire à son ascension. La prise en compte de cette dimension sociologique instaure un point de rencontre entre les facteurs hétérogènes et leur équivalent endogènes, sur lesquels nous allons nous pencher dans la section qui suit. En effet, Tinayre a déployé sa connaissance du milieu petit-bourgeois pour décrire dans ses textes la position des femmes des classes urbaines moyennes dans la France des années 1890-1930. Dans nombre de ses romans et tout particulièrement au début de sa carrière, elle s'est penchée sur les questionnements qui animaient ses contemporaines, leur aspiration à l'égalité, leur désir d'émancipation, en particulier par le biais du travail, la dénonciation du « devoir conjugal » et de l'emprisonnement auquel les conduisait souvent le mariage, le poids des préceptes religieux. Si l'activité fictionnelle est ici mise au service d'une thématique sentimentale, elle permet également de plonger le lecteur/la lectrice dans l'univers de la petite bourgeoisie parisienne au féminin. L'écriture tinayrienne puise donc concomitamment au registre traditionnel du roman d'amour et à celui plus original, pour l'époque, et certainement plus subversif, du roman social. Or, les décideurs - éditeurs, critiques, politiques - rechignaient à promouvoir ce qui constituait implicitement à la fois une remise en question du modèle établi, c'est-àdire des normes patriarcales, et une transgression. Compte tenu des contraintes imposées à Tinayre, du fait de son identité genrée, il lui était encore plus difficile qu'à ses confrères d'exercer le moindre contrôle sur la production et la diffusion de ses écrits. Dépendante économiquement des revenus que lui assurait la publication de ses romans - chez les Tinayre, c'est madame qui subvenait aux besoins de la famille elle ne pouvait que moduler son discours. C'est ainsi qu'avant la parution de $\mathrm{La}$ Rançon en 1898, son éditeur, Calmann-Lévy, lui demande de : «Biffer au passage toute expression trop libre. Supprimer toute analyse trop hardie, toute page risquée (scènes conjugales et extraconjugales indiscrètes). [...] Se borner à indiquer les faits toutefois que le détail sera inadmissible pour la lecture en famille » (Grenaudier-Klijn 49). Il n'est pas impossible de penser que, si Tinayre avait pu persister dans la dénonciation d'une société foncièrement injuste envers les femmes de la petite et 
moyenne bourgeoisie, ses premiers romans seraient sans doute davantage lus aujourd'hui et occuperaient une place plus visible dans le canon.

Enfin, les romans tinayriens sont difficilement situables d'un point de vue générique, ce qui là encore rend leur classification moins aisée et complique donc leur insertion dans le canon. À l'analyse des textes, on constate ainsi une grande variété thématique, notre romancière s'étant intéressée à d'autres nations - la Norvège, la Turquie - et à d'autres époques - l'histoire antique, le Grand siècle, les années 1830. Certains de ses romans trouvent donc leur place dans les catégories «roman historique », «roman régional» ou encore «roman de voyage», genres souvent marginalisés par le processus de canonisation car jugés moins «nobles ». D’autres empruntent à deux genres - le roman d'amour et le bildungsroman. Mais là encore, le canon opérant selon un ordonnancement taxinomique précis, le caractère fluide propre au roman tinayrien se révèle un handicap. De plus, aucun des genres auxquels emprunte Tinayre n'est particulièrement novateur, même si, dans le cas du roman d'amour et du roman d'initiation, l'on doit lui reconnaître d'avoir fait fusionner deux traditions généralement considérées comme antithétiques. Or, comme l'explique Pierre Bourdieu, le canon a toujours favorisé les avant-gardes.

\section{Manque d'audace}

Nonobstant ces présupposés, d'autres facteurs, endogènes ceux-là, peuvent nous aider à comprendre l'oubli imposé à l'œuvre de Tinayre. D'un point de vue purement esthétique, l'écriture tinayrienne n'est pas suffisamment novatrice. Elle ne manifeste ni la pétulance, l'originalité créative, la sensualité de Colette, par exemple, ni encore l'exorbitance de Rachilde. Son vocabulaire, sa grammaire, sa syntaxe, demeurent en outre foncièrement pré-modernes, et ne débouchent pas sur une véritable transformation du langage, même si James Joyce a loué l'originalité de $L a$ Maison du péché (1903) et l'art avec lequel Tinayre avait exploité la technique du «stream of consciousness » et confronté le discours de la sexualité, de l'art et de la vie à celui de l'église (Kershner 285).

Si le nom de notre auteure n'est que rarement mentionné dans les annales, y compris dans les anthologies d'obédience féministe, c'est aussi parce que le féminisme tinayrien ne peut être taxé, en toute honnêteté, que de frileux. Là encore, Tinayre vacille entre deux pôles. Elle fait montre d'un indubitable conservatisme dans sa conception de «femme[s], très femme $[\mathrm{s}]^{6}{ }^{2}$ qui ne cessent d'aspirer à l'union avec 
un «maître » dont la supériorité intellectuelle et morale ne saurait être remise en question, tout en n'hésitant pas parallèlement à dénoncer l'argutie du discours sexuel en vigueur et à revendiquer l'autonomie et la légitimité du désir féminin : «Moi, m'écriai-je [il s'agit de son premier roman, Avant l'amour], je ne renonce à rien. [...] Je vivrai la vie... J'ai droit à l'amour... Si je ne puis le trouver dans le mariage... alors » (Avant, 101-102). Le discours normatif de l'hétérosexualité que prône Tinayre, son positionnement idéologique, concomitamment ancré dans le culte de la féminité et partisan d'une hétérogénéité radicale, positionnement qui, de surcroît, se durcit singulièrement dans les vingt dernières années de sa vie, est en contradiction autant avec l'idéal féministe qu'avec l'idéal universaliste. Tinayre dresse un constat sans jamais réellement bousculer, moins encore transformer la position de ses contemporaines. Prenant ouvertement la défense des femmes aux premiers temps de sa carrière, elle semble tourner casaque et adopter vis-à-vis de celles-ci un discours infiniment plus édulcoré où affleure parfois une forme de condescendance. Même si les silences, les absences et les lacunes ont valeur d'enseignement, ces atermoiements compliquent la position des féministes vis-à-vis de l'héritage tinayrien. Surtout, l'ambivalence du discours dément toute authentique prise de position, contribuant ainsi à auréoler l'œuvre en général d'une aura de pusillanimité s'accordant mal avec l'inscription au canon.

Enfin, elle fit souvent preuve de maladresse, mésestimant les réactions de la presse et du public lorsqu'elle refusa la Légion d'honneur en 1908, au motif qu'elle aurait eu l'air d'une cantinière, et se retrouvant impliquée quinze ans plus tard dans le scandale du Prix Flaubert. Sa trajectoire, au sens où l'entend Bourdieu, s'apparente à une succession d'atermoiements, d'hésitations, d'oscillations entre subversion et conservatisme, qui contribue à donner de Tinayre l'image d'une romancière trop souvent assujettie à la satisfaction de ses intérêts personnels immédiats au détriment d'un authentique investissement dans son travail d'écriture.

\section{Omission ou exclusion ?}

Que conclure de tout cela ? Y a-t-il eu injustice ? Marcelle Tinayre mérite-telle de figurer au canon littéraire français? La réponse ne peut qu'être mitigée. Femme, petite-bourgeoise, très à droite à la fin de sa vie - jusqu'en 1944, elle publia un billet hebdomadaire dans Voix françaises, « une revue [fondée en 1941] clairement située dans la droite pétainiste »(Quella-Villéger 442$)^{7}$ - Tinayre s'inscrit dans un 
champ de déterminations historiques peu favorables à son passage à la postérité. Il est certain que Tinayre n'a pas su aller assez loin, et il est tout à fait possible que l'énergie, le talent et la curiosité intellectuelle nécessaires à une exploration plus approfondie des possibilités que lui offrait l'hybridité générique, par exemple, lui aient manqué. Aurait-elle su développer de manière plus novatrice les intrigues et les protagonistes qu'elle avait conçus, peut-être aurait-elle été en mesure de parvenir à une écriture véritablement originale. Mais là encore, on ne peut sous-estimer les difficultés auxquelles une telle audace l'aurait confrontée. Certes, ce risque existait aussi pour les écrivains. Mais étant donné les circonstances hors-textuelles dans lesquelles il opérait, l'acte d'écrire demeurait forcément plus radical, plus subversif pour une femme que pour un homme, ce que nous avons pu constater aux réactions de son éditeur à l'occasion de la parution de La Rançon, son deuxième roman. Encore une fois, nous ne pouvons ignorer le poids des a priori doxiques sur la création, la diffusion et la réception des œuvres écrites. Freinée par le contexte idéologique propre à l'aire culturelle particulière à laquelle elle participait, Tinayre était prise sous une double contrainte : satisfaire son éditeur, et, dans une moindre mesure, son public, ou aller au bout de son propos. Elle décrit ce dilemme dans La Rebelle : « [...] ce n'est pas dans Le Monde féminin ${ }^{8}$ que Josanne pourra exprimer, sincèrement, ses opinions... Monsieur Foucart [le rédacteur en chef] exige que la charité soit discrète, la misère voilée, et que la douleur et la mort mêmes gardent un «petit air parisien » (99). Sans minimiser le schématisme un brin facile des constructions romanesques auxquelles Tinayre a souvent recours, son œuvre n'en recèle pas moins une originalité thématique certaine. Elle a écrit sur le désir féminin, créé des héroïnes ne se satisfaisant pas de la seule maternité, revendiqué le droit au travail et l'indépendance économique des femmes, et dénoncé l'hypocrisie des conventions bourgeoises. Elle est même allée jusqu'à évoquer dans La Rebelle en 1905 une tentative d'avortement dont on s'étonne que les censeurs l'aient laissé passer. Même si, à bien des égards, le roman tinayrien peut être considéré comme désuet, la dimension sociologique de ses textes est à elle seule méritoire. Si redécouverte il y a un jour, ce sera sans doute au titre de la contribution apportée par une activité fictionnelle mise au service d'un groupe socio-économique précis, qui donne à voir la réalité de l'existence des femmes de la petite et moyenne bourgeoisie française des premières années du $\mathrm{XX}^{\mathrm{e}}$ siècle. Cette valeur de témoignage devrait, à elle seule, encourager la relecture et la 
réhabilitation de ses textes, car ce n'est pas dans les romans de ses contemporains que l'on sera en mesure de (re)découvrir ce que fut la réalité de ces femmes.

Pour analyser de manière exhaustive l'évolution du parcours d'un/e écrivain/e - illustré ici par la trajectoire de Marcelle Tinayre - allant de la production initiale à la pérennisation, il est nécessaire de mêler aux «interprétations internes » focalisées exclusivement sur les caractéristiques formelles des textes, les «explications externes » (Bourdieu, Raisons pratiques, 62) tenant compte des déterminations historiques au sein desquelles émergèrent ces textes. En développant la théorie du champ, Bourdieu démontre de manière convaincante qu'il n'est plus possible de sousestimer l'interdépendance des facteurs hétérogènes et endogènes et l'influence que ceux-ci exercent sur notre propension à préserver ou rejeter des annales telle ou telle œuvre. Ce n'est qu'ainsi, me semble-t-il, que nous parviendrons à une lecture multidirectionnelle du système canonique français, susceptible de nous amener à une appréciation plus juste, plus équilibrée et peut-être plus constructive, de son mode de fonctionnement. Il ne me semble pas foncièrement injuste que le nom de Marcelle Tinayre ne figure pas au panthéon de la littérature française. Je suis moins convaincue toutefois qu'il s'agisse là d'une simple omission, et aurais tendance à penser que le doigt de l'exclusion s'est glissé dans l'engrenage.

\section{Ouvrages cités}

André, Sylvie. «Pistes pour repenser les études littéraires au $\mathrm{XXI}^{\mathrm{e}}$ siècle ». Diogène 229-230 (janvier-avril 2010) : 109-125.

Billy, André. L’Époque 1900. Paris : Tallandier, 1951.

Bourdieu, Pierre. «Pour une science des œuvres ». Raisons pratiques : Sur la théorie de l'action. Paris : Seuil, 1994. 61-89.

---. Le Sens pratique. Paris : Minuit, 1980.

--- et Jean-Claude Passeron. La Reproduction. Éléments pour une théorie du système d'enseignement. Paris : Minuit, 1970.

Charasson, Henriette. «La littérature féminine ». Vingt-cinq ans de littérature française. Tableau de la vie littéraire de 1895 à 1920, tome II. Dir. Eugène Montfort. Paris : Librairie de France, 1925.

Chardenet, Patrick. «Éditorial. L'avenir des langues internationalisées ». Le Français à l'université 1 (2011) : 1 et 9. 
Grenaudier-Klijn, France. Une Littérature de circonstances. Texte, hors-texte et ambiguïté générique à travers quatre romans de Marcelle Tinayre. Berne : Lang, 2004.

Havet, Mireille. «Visite à Marcelle Tinayre ». Les Nouvelles littéraires (3 décembre 1922) : n. p.

Kershner, R. B. Joyce, Bakhtin, and Popular Literature. Chronicles of Disorder. Chapel Hill : The U of North Carolina P, 1989.

Lavaud, Martine. «Ratés, oubliés et minores : sur quelques fictions excentriques de l'histoire littéraire au $\mathrm{XIX}^{\mathrm{e}}$ siècle ». Fictions d'histoire littéraire. Dir. JeanLouis Jeannelle. Rennes : PU de Rennes, 2009. 157-169.

Quella-Villéger, Alain. Belles et rebelles. Le roman vrai des Chasteau-Tinayre. Bordeaux : Aubéron, 2003.

Slama, Béatrice. «De la 'littérature-féminine' à 'l'écrire-femme' : différence et institutions ». Littérature 44 (1981) : 51-71.

Tinayre, Marcelle. Avant l'amour. Paris : Calmann-Lévy, 1897.

---. La Rebelle. Paris : Calmann-Lévy, 1905.

Trintignac, Pierre. «Les écrivains de chez nous: $\mathrm{M}^{\mathrm{me}}$ Marcelle Tinayre». Le Courrier du centre (30 août 1934) : n. p.

Von Kulessa, Rotraud. Entre la reconnaissance et l'exclusion. La position de l'autrice dans le champ littéraire en France et en Italie à l'époque 1900. Paris : Champion, 2011.

\section{Notes}

1 Voir par exemple France Grenaudier-Klijn, Une Littérature de circonstances. Texte, hors-texte et ambiguïté générique à travers quatre romans de Marcelle Tinayre. Bern : Lang, 2004 ; Mélanie Collado, Colette, Lucie Delarue-Mardrus, Marcelle Tinayre. Émancipation et résignation. Paris, L'Harmattan, 2003 ; Alain Quella-Villéger, Belles et rebelles : le roman vrai des Chasteau-Tinayre, Bordeaux : Aubéron, 2000. Elizabeth Ceaux, «Voyage dans le passé avec Marcelle Tinayre », Quaderni di filologia e lingue romanze 1 (1986) : 221-243 et « La femme au début du XX $\mathrm{X}^{\mathrm{e}}$ siècle à travers les romans de M. Tinayre », Quaderni di filologia e lingue romanze 6 (1984) : 206-220 ; Gabrielle Houbre, «L'honneur perdu de M. Tinayre: l'affaire de la légion d'honneur ratée (1908) », Les Ratés de la littérature, dirs. Jean-Jacques Lefrère, Michel Pierssens et Jean Didier Wagneur, Tusson, Charente : Du Lérot, 1998: 89-102. Voir aussi Diana Holmes, Women in Context : French Women's Writing 1948-1994, Londres, Athlone, 1996 et Jennifer Waelti-Walters, Feminist Novelists of the Belle Epoque, Bloomington : Indiana UP, 1990.

2 Apprenant que le nom de Céline figure au recueil des célébrations nationales 2011, Serge Klarsfeld, président de l'association des fils et filles de déportés juifs de France (FFDJF), en demande le retrait immédiat. Le ministre de la Culture, Frédéric Mitterand, décide de retirer l'écrivain de la liste.

3 À cet effet, je renvoie lecteurs et lectrices à l'introduction de Nathalie Morello et Catherine Rodgers dans Nouvelles écrivaines : nouvelles voix ? Amsterdam : Rodopi, 2002, pp. 7-45. 
4 L'anthologie est parue en 2011. On peut apprendre sur le site Internet de l'éditeur que : "With equal attention to all genres, historical periods and registers, this is the most comprehensive guide to literature written in French ever produced in English, and the first in decades to offer such an array of topics and perspectives."

http://www.cambridge.org/gb/knowledge/isbn/item5705311/?site_locale=en_GB. Téléchargé 19 août 2011.

[Accordant la même attention à tous les genres, registres et périodes historiques, cette anthologie de littérature française en anglais est la plus complète qui soit, et la première depuis des décennies à proposer une telle gamme de thèmes et de perspectives.]

5 Rappelons que pour être membre de la SDGL, il ne suffisait pas d'être écrivain/e ; il fallait être publié/ée.

6 Il s'agit d'une référence à La Rebelle, p. 8.

7 La place nous manque ici, mais il serait pertinent d'examiner les raisons susceptibles d'avoir contribué à un tel durcissement idéologique. Contentons-nous pour le moment de suggérer que ce virement à droite fut probablement favorisé d'une part par les engagements vichystes de son dernier compagnon, Noël Pinelli - thèse que défend Alain Quella-Villéger - d'autre part par l'évolution d'un féminisme de plus en plus axé sur la famille, qui l'amena à collaborer au journal Le Flambeau, organe du parti des Croix de Feu auquel succéda le Parti social français (PSF). Voir Kevin Passmore, «Planting the Tricolor in the Citadels of Communism : Women's Social Action in the Croix de feu and Parti social français. » The Journal of Modern History 71-4 (décembre 1999) : 814-851.

8 Il s'agit du magazine pour lequel travaille Josanne Valentin, l'héroïne de La Rebelle. 